\title{
Role of Systemic Inflammation in Neurodegenerative Diseases
}

\author{
Beatrice Duvert and Alireza G. Senejani* \\ Department of Biology and Environmental Science, USA
}

\section{Abstract}

The role of systemic inflammation in neurodegeneration is complex. It involves interplay between several pro-inflammatory and anti-inflammatory factors, DNA damage and a cycle of neurotoxic inflammation and tissue degradation. Neuroinflammation can trigger neurodegeneration. Though suppressing systemic inflammation might be assumed to be neuroprotective against neurodegeneration, inhibiting certain features of the systemic immune response may cause damage.

Introduction

In this review we briefly cover the following topics:

- Role of systemic inflammation on microglia activation

- Involvement of systemically generated anti-brain antibodies

- Neuron-microglia interactions downregulate neuroinflammation

- Maladaptive microglial priming provokes neuroinflammation

*Corresponding author: Alireza G. Senejani, Department of Biology and Environmental Science, USA

Submission: 酒 March 18, 2019

Published: :

Volume 1 - Issue 5

How to cite this article: Beatrice Duvert and Alireza G. Senejani. Role of Systemic Inflammation in Neurodegenerative Diseases. J Biotech Biores.1(5). JBB.000525.2019.

Copyright@ Alireza G. Senejani, This article is distributed under the terms of the Creative Commons Attribution 4.0 International License, which permits unrestricted use and redistribution provided that the original author and source are credited.
- Energetic cost of systemic inflammation may inhibit neuron metabolic dependent maintenance

- The role of pathogen infections in neuroinflammation

- The DNA damage response triggers neuroinflammation

- Mitochondrial DNA Damage Triggers Neuroinflammation

- Immune cell recruitment to the CNS regulates inflammation

- Neuroinflammation regulation dependent on distinct macrophage populations

- NF-kB and IL-1B as proinflammatory targets for neuroinflammation

\section{Role of systemic inflammation on microglia activation}

Normal activity of microglia native to the Central Nervous System (CNS) is responsible for routine immune surveillance activities that normally proceed without generating an inflammatory response. These activities include phagocytosis of apoptotic cells and cell debris as well as synaptic pruning [1]. Microglia can also serve important roles in neural growth and generating cell survival signals to allow proper tissue maintenance and renewal [2]. However, an imbalance of microglial hyper-activation and termination may lead to a neurotoxic cycle of neural damage in response to an overproduction of proinflammatory factors and Reactive Oxygen Species (ROS) [2,3].

Prolonged local cytotoxic inflammation advances neurodegeneration progression through axon injury, demyelination, and other nerve cell damage [1,2]. Systemic inflammatory mediators can disrupt the homeostasis responsible for neuron and microglial cell health [1]. Though the microenvironment of the brain is mostly protected by the Blood Brain Barrier (BBB), it is not completely impenetrable to the effects of systemic inflammation. Inflammatory cytokines from the periphery can interact with macrophages in highly vascularized sensory circumventricular organs where the BBB is less tightly regulated $[4,5]$.

Further communication can occur through cerebral endothelial cells signalling perivascular macrophages, which can then signal microglia in the CNS microenvironment 
[4]. Furthermore, systemic inflammation can induce a pathological weakening of the tight junctions established between the astrocytes and endothelial cells that form the BBB. Infiltration of peripheral inflammatory cytokines and immune cells can lead to neurotoxicity in the brain environment and can also activate microglial cells towards proinflammatory activities. In an experimental model of sepsis, injection of Lipopolysaccharide (LPS), an endotoxin used to mimic systemic inflammation by immune response induction, switches microglia to a proinflammatory state [1].

Systemic inflammation induction was followed by an increase in gene expression for inflammatory mediators including IL-1B, IL-6, TLR2, and TLR 4 and an increase in microglial activation four hours post injection [1]. LPS injection in animal prion disease models also results in macroglia switching to a hyperactive proinflammatory phenotype producing IL-1B and TNF cytokines and increased neuron apoptosis [6]. Proinflammatory microglia switching was also found in a Parkinson's disease model, in which LPS injection exacerbated disease progression [7].

Normal aging results in a decline of adaptive immunity while innate immunity remains intact creating a persistent proinflammatory state in tissues. This contributes to an age-related shift of microglia from a resting or downregulated state to a primed state that is more easily activated in response to immune threats [4]. Several autoimmune diseases and neurodegenerative diseases also contribute to a proinflammatory state of the brain [1].

\section{Involvement of systemically generated anti-brain anti- bodies}

Cognitive impairment is seen in chronic autoimmune disorders such as Systemic Lupus Erythematosus (SLE) where systemic inflammation persists. Systemically generated anti-brain antibodies that enter the CNS when the Blood Brain Barrier (BBB) becomes leaky have been detected in SLE. N-Methyl-D-Aspartate (NMDA) glutamate receptors are instrumental in neuron synaptic plasticity in the hippocampus [1]. Cross-talk between anti-brain antibodies and NMDA receptors is associated with cognitive decline as high levels of these antibodies can lead to decreased availability of these receptors through mechanisms such as cross-linking and receptor internalization.

In certain cases of SLE, anti-brain antibodies have been found to promote the open conformation of NMDA receptors causing an increase in generation of postsynaptic potentials and in rodent models has been shown to cause hippocampal neurodegeneration [1]. A study was conducted on patients with mild cognitive impairments and dementias including Alzheimer's disease to evaluate the presence of NMDA receptor antibodies and leaky Blood-Cerebral Spinal Fluid-Barrier (B-CSF-B) markers. NMDA receptor antibodies and B-CSF-B dysfunction markers were present in the serum of patients and age-matched healthy controls and associated with aging [8].

Though B-CSF-B dysfunction markers were elevated in dementia patients versus controls, NMDA receptor antibodies were not statistically different. However, a sub-group of patients with "Non-Classical" $\mathrm{AD}$ with at least one characteristic of $\mathrm{AD}$ at initial diagnosis were compared to patients with classical AD. The non-classical AD group had statistically significant elevated levels of NDMA receptor antibodies and B-CSF-B dysfunction and follow up studies found that increased levels were associated with poorer prognosis and cognitive decline compared with classical AD patients [8].

\section{Neuron-microglia interactions downregulate neuroin- flammation}

Microglia are native to the CNS and exist in a less inflammatory state than systemic immune cells. This downregulated inflammatory state is stimulated by normal cross-talk between neurons and microglia. For instance, neurons release neurotropic factors that suppress the expression of MHC II genes in microglia [4]. Microglia have many neurotransmitter receptors making them susceptible to anti-inflammatory regulation from neurons which may secrete, for example, $\gamma$-Aminobutyric Acid (GABA) which activates GABA receptors and in turn inhibits Nuclear Factor-Kappa B (NF-kB) production. Direct ligand binding of membrane proteins on neurons to their microglial receptors can also promote microglial downregulation by recruiting SHP-1 and SHP-2 phosphatases that inhibit intracellular immune signalling.

\section{Maladaptive microglial priming provokes neuroinflam- mation}

Dysfunctional microglial activity is mediated by impaired neuron-microglia interactions. Repetitive microglial inflammatory priming from sustained systemic inflammation can exacerbate neurodegenerative disorders. Neuron loss can destabilize the important immunoregulatory interactions in microglia, and in this way, neurodegeneration can further contribute to a toxic cycle of neuroinflammation [4]. In a mouse model for prion disease, LPS injection increases the expression of microglial activating receptors making them more responsive to activation during amyloid beta exposure [9]. Another prion disease model showed that microglial cells are primed for increasingly severe inflammatory responses through systemic inflammation activation [6].

\section{Energetic cost of systemic inflammation may inhibit neuron metabolic dependent maintenance}

Part of the burden of systemic inflammation on neurons is proposed to come from energetic depletion of a costly immune response which may sequester energy sources from oligodendrocytes responsible for neuronal network maintenance [1]. In addition to generating the myelin sheaths surrounding axons, oligodendrocytes help support axonal metabolic function, likely by providing neurons access to lactate produced through glycolysis $[10,11]$. Lactate is exclusively transported by Monocarboxylate Transporters, and Monocarboxylate Transporter 1 (MCT1) is particularly abundant in oligodendrocytes [10].

MCT1 inhibition in vitro results in neuron loss, specifically mediated by impaired lactate secretion; and MCT1 deficient mice show axon degeneration and other morphological signs of neurodegenerative disease [10]. Furthermore, patients with Amyotrophic Lateral Sclerosis (ALS) have impaired oligodendrocyte function and reduced MCT1s [10,12]. Without a constant energy 
supply to support energetically costly axonal maintenance, neural connectivity is impaired, which could lead to cognitive decline symptomatic in neurodegeneration [1]. In certain cases of systemic inflammation, vasculature and tissue perfusion are impaired.

It could reduce the supply of energy needed by the oligodendrocytes to provide glycolysis mediated feeding of the axons through lactate. Inadequate vascular function associated with a prolonged inflammatory response is observed in both sepsis and in autoimmune diseases like antiphospholipid syndrome; and patients with both conditions experience cognitive impairment [1]. Physical exercises are reported to potentially play an important role in epigenetic regulation of gene expression positively influencing inflammation and neurodegenerative disease [13].

\section{The role of pathogen infections in neuroinflammation}

Systemic inflammation can wreak havoc on highly complex brain architecture and wiring. Preserving brain architecture is often placed at a high enough priority that the brain is tolerant in certain cases to co-exist with pathogens that manage to cross the BBB. This may be favoured rather than mounting an immune response to eradicate the infection, which could cause severe tissue damage and irreparably alter brain architecture. The herpes simplex virus and Cryptococcus gattii have been found to reside in the brain without triggering an immune response [1].

Infectious agents can trigger neuroinflammation and Alpha-Synuclein protein ( $\alpha$-Sync), the pathological hallmark of Parkinson's disease [14]. Microglia express receptors for and can produce IL-10, an immunosuppressive cytokine, making it possible for them to regulate their own inflammatory response within the CNS after activation. Borrelia burgdorferi or Neisseria meningitidis were found to induce murine microglia to produce IL-10, which inhibited the microglial proinflammatory cytokine production that is initiated after initial pathogen exposure. This demonstrates a microglia mediated mechanism for pathogen tolerance during chronic infections [15].

Though pathogens can sometimes exist asymptomatically in the brain, they may cause harm contributing to neurodegeneration. For instance, there is evidence that infections of the brain are associated with neurodegenerative diseases such as Alzheimer's [16]. As part of the innate immune host defence, macrophages, including microglia, phagocytize pathogens and trigger the inflammasome. The inflammasome releases caspase 1, which activates IL-1B as part of the proptosis programmed cell death pathway [1,17]. Proptosis results in release of cellular contents after lysis of the phagocytotic cells [17].

These released cytosolic contents include the engulfed active bacteria and inflammatory signals that recruit secondary phagocytotic neutrophils to destroy the pathogens $[17,18]$. In a process known as the "Respiratory Burst", neutrophils increase their oxygen consumption and through the NADPH oxidase pathway, stimulate superoxide ROS release, which has an antimicrobial effect [17-19]. However, an overproduction of ROS can also damage surrounding tissue, and could be instrumental in creating oxidative DNA damage that may lead to neuron apoptosis $[18,19]$. Intracellular NOD-Like Receptors (NLR) in microglia can detect microbial agents that have entered host cells [20].

Therefore, in addition to further stimulation of the innate immune response to eradicate invading pathogens, microglia through NLR stimulation are important in innate-adaptive immunity cross talk and stimulating recruitment of the adaptive immune response [20]. This recruitment may be crucial to downregulating the pro-neuroinflammatory response and preventing neuroinflammation [20,21]. The long-term dynamics of host-pathogen responses during prolonged chronic infections warrant investigation to elucidate what affect changes in tolerance may have on immune response and neurodegeneration.

\section{The DNA damage response triggers neuroinflammation}

The DNA Damage Response (DDR) also has an important role to play in neuroinflammation. The Ataxia Telangiectasia Mutated (ATM) DDR protein is a crucial player in DDR signalling. ATM has specific importance for neuron genomic stability playing a role in recruitment of DDR proteins to the location of DNA damage and supporting other DDR activities such as H2AX histone phosphorylation. However, ATM in coordination with p53 is also responsible for stimulating NF-kB activation, which promotes neuroinflammation [22].

In addition to activating ATM and p53, the DDR activates ATMIKK/-Interferon (IFN)- signalling and subsequent downstream activation of interferon-inducible myeloid differentiation transcriptional activator (IFI16) stimulating inflammatory cytokine production of IL-1, IL-18, and others [22]. A study on the neurodegenerative disease Friedreich's Ataxia (FA) provides useful insight into the role of DNA damage on microglial activation. The frataxin protein is involved in mitochondrial formation of IronSulphur complexes that serve as important protein cofactors for cellular functions including electron transport [23].

Deficient frataxin protein in a FA mouse model results in DNA damage, which activates DNA damage repair genes MUTYH and PARP-1 specifically in the microglia stimulating microglial activation. More microglia were in the active state in FA mice compared with wild type mice after LPS injection. Suppressing these genes may be a way to suppress microglial activation and therefore mitigate a neurotoxic inflammatory response that can progress into neurodegenerative disease [24].

\section{Mitochondrial DNA damage triggers neuroinflammation}

Aging of microglia in the CNS is another important factor of neurodegeneration. Accumulation of mtDNA damage in the microglia increases ROS levels, which in turn stimulate the release of inflammatory factors like NF-kB and the proinflammatory cytokine IL-1B resulting in damaging neuroinflammation. Mitochondrial Transcription Factor A (TFAM) can either play a role in mtDNAstabilization or serve to stimulate a proinflammatory response depending on where it is localized. When localized in intact microglia cells, TFAM can help stabilize mtDNA damage preventing an overproduction of ROS and neuroinflammation [25].

However, when TFAM is secreted from necrotic cells, it can trigger an immune response. Lower levels of IL-1B positive cells were observed in transgenic mice overexpressing TFAM 
compared with wild type mice after exposure to LPS. Also, though levels of the 8-Oxo-Gua DNA damage marker were mostly colocalized with these IL-1B positive microglia, fewer were found in the TFAM overexpressed mice. LPS injection resulted in IL-1B positive microglia mainly localized to the hippocampus including the Sub-Ventricular Zone (SVZ). Since the SVZ is an important site of neural stem cell proliferation and possible renewal after neurodegenerative episodes, the role of TFAM and mtDNA damage maintenance is important as it can affect function in this crucial neurogenesis center [25].

\section{Immune cell recruitment to the CNS regulates inflammation}

Proper immune system function at both the systemic and local level is important for preventing neurodegeneration (Figure 1) [26]. Recruitment of CD4+ T cells and peripheral monocytes regulate inflammation in the CNS. The suppression of this immune cell recruitment when patients are on anti-inflammatory drugs may be a potential factor explaining mixed results of immunosuppressive drug regimens either preventing neurodegeneration or failing to do so when used as a treatment regimen for neurodegenerative diseases such as Alzheimer's [2].

It has been proposed that the changing roles of microglia native to the CNS and infiltrating monocyte-derived macrophages from the peripheral blood system throughout different phases of neurodegenerative disease progression are responsible for different neuroprotective response outcomes [2]. These dynamic roles are important considerations for the proper application of immunosuppressive therapies for neurodegeneration.

In addition to the role microglia play in surveilling the CNS for potential threats, infiltrating monocyte-derived macrophages are also important for amyloid-beta deposit elimination as well as insulin growth factor secretion to stimulate neural cell renewal to counter the suppression of insulin growth factor after TNF activation from the inflammatory response. A systemic immune response to local cytotoxic inflammation is important for interrupting the neurotoxic cycle. Thus, immunosuppressive drug treatment may inhibit important systemic immune system activity during the phase where the reparative immune cells are needed to terminate local inflammation [2].

However, anti-inflammatory treatment may be appropriate for earlier phases of inflammation before local inflammation becomes neurotoxic [2,27]. A protective autoimmune response mediated by CD4+ T cells may be important in this immune response shift after acute CNS injury. This is supported by findings showing that $\mathrm{T}$ cell vaccination of an $\mathrm{AD}$ mouse model slows disease progression and in patients with $\mathrm{AD}$, deficiencies with monocytes differentiating into macrophages have been observed as well as deficient amyloidbeta clearance effectiveness by infiltrating macrophages [2].

Infiltrating monocyte-derived macrophages have also been found instrumental in spinal cord injury repair in mice. Suppressing macrophage infiltration lead to deficient recovery while increasing the monocyte population in the peripheral blood resulted in enhanced recovery. During spinal cord injury, native microglia initiate an inflammatory response while infiltrating macrophages express IL-10 promoting an anti-inflammatory response at the spinal cord injury site [28]. Results of a recent study show peripheral exposure to spinal cord antigens is associated with spinal cord and brain inflammation leading to degeneration of CNS cells [29].

Infiltrating monocyte-derived macrophages play an important role in mitigating local inflammation and promoting cell renewal as has been observed following decreased inflammatory responses to retinal injury. Increasing the level of monocytes in the peripheral blood also increased the survival of the retinal ganglion cells after injury and conversely, blocking infiltration reduced survival [30].

\section{Neuroinflammation regulation dependent on distinct macrophage populations}

Diversity in macrophages native to or recruited to the CNS may also be important to autoimmunity in the CNS. There are proinflammatory macrophages with associated markers such as Ly6C+ and anti-inflammatory macrophages with associated markers such as Ly6C-, which are important during the resolution phase of inflammation. An experimental autoimmune model of the ocular system was used as model for CNS autoimmunity. Different macrophage phenotypes and functions were observed throughout the different phases of the inflammatory disease progression [31].

The level of macrophages with anti-inflammatory functions rose throughout the disease course while the level of macrophages with inflammation associated markers like Ly6C+decreased. Also observed was disease prevention when macrophage infiltration was blocked at the onset of the disease while blocking infiltration at the peak of the disease resulted in less anti-inflammatory macrophages in the retina and worse progression. This indicates that the kinetics of distinct macrophage population levels is crucial in disease progression [31].

\section{NF-kB and IL-1B as proinflammatory targets for neuroinflammation}

In a model for Huntington's disease, NF-kB was shown to induce apoptosis by contributing to c-Myc and p53 induction after administration with a kainic acid excitotoxin. The kainic acid agonist for the glutamate responsive kainite receptor (KA) was found to stimulate neuron cell death [32]. Though NF-kB is normally sequestered to the cytoplasm, its activation and translocation to the nucleus induces c-Myc and p53 expression, which subsequently stimulates apoptosis. Blocking NF-kB translocation was found to decrease c-Myc and p53 induction as well as DNA fragmentation. These findings also suggest that blocking KA receptors may be neuroprotective in neurodegenerative diseases [32].

The important role of NF-kB in neuron apoptosis is also supported by a study showing NF-kB inhibition decreases apoptosis after ninety minutes of cerebral hypoxia but not with prolonged hypoxia more than two hours when necrosis is considered more of a contributing factor to cerebral injury. In a Parkinson's disease model where systemic inflammation was induced, IL-1B inhibition reversed disease progression leading to an increase in dopaminergic 
neurons after treatment providing support that targeted inhibition of this cytokine could be a useful neurodegenerative disease treatment. This disease reversal was not associated with any changes in microglial activation indicating that the IL-1B inhibition mediated activities downstream of microglial activation [7].

\section{Conclusion}

The sources and different factors of CNS microglial activation, amplification, and termination are important in order to develop treatments promoting a well-modulated and maintained systemic immune system response (Figure 2). Not only are internal factors such as DNA damage in microglial cells of the CNS important, but the infiltration of macrophages from the periphery of the local neuroinflammation site is also crucial to avoiding a cycle of neurotoxicity and neurodegeneration resulting from neuroinflammation triggered by pathogens, amyloid beta deposits, acute tissue injury, and other factors (Figure 3). When considering possible treatment regimens, targeted inhibition of inflammation might be key instead of broad-spectrum immune suppressive therapies to combat systemic inflammation associated with neurodegenerative diseases (Figure 1-3).

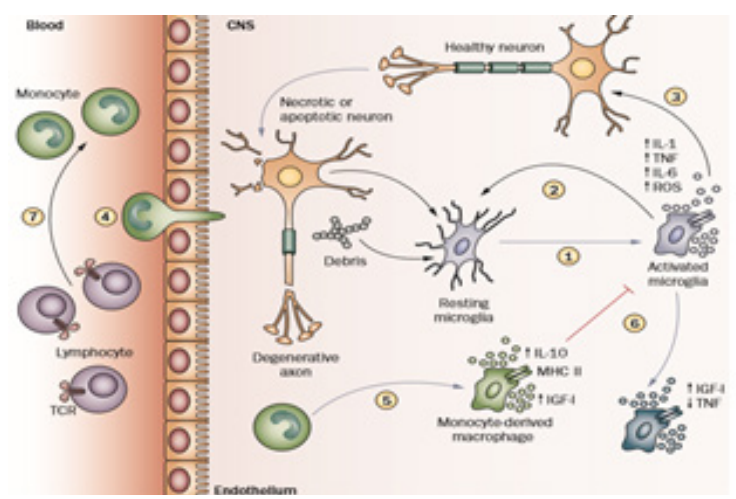

Figure 1: Immune cells in neurodegenerative conditions. Black arrows highlight relationships between cells (and other factors), while blue arrows indicate cellular conversions. Dying cells and/or the accumulation of debris or aggregated proteins activate resting microglia in the CNS.

1. Activated microglia phagocytose debris and aggregated proteins while concurrently secreting toxic compounds, including pro-inflammatory cytokines (such as iL-1, TNF and iL-6) and ROS. if the microglial response is uncontrolled or prolonged, these toxic agents contribute to the development of a self-sustaining neurotoxic cycle that involves further activation of naive microglia.

2. Loss of surrounding neurons.

3. At this stage (equilibrium stage), assistance from the systemic immune system becomes essential to control local inflammation. Blood derived monocytes infiltrate the damaged parenchyma.

4. Differentiate locally into macrophages.

5. These cells efficiently engulf debris, secrete growth factors and anti-inflammatory cytokines, and suppress microglial activation.

6. Under neurodegenerative conditions, resident microglia are unable to provide these essential functions. T-helper lymphocytes (mainly CD4+ T cells) augment the recruitment of blood monocytes to the CNS.

7. Indirectly regulate the phenotype of microglia.

Abbreviations: IGF-I: Insulin-Like Growth Factor I; IL: Interleukin; ROS: Reactive Oxygen Species; TCR: T Cell Receptor; TNF: Tumor Necrosis Factor [1].

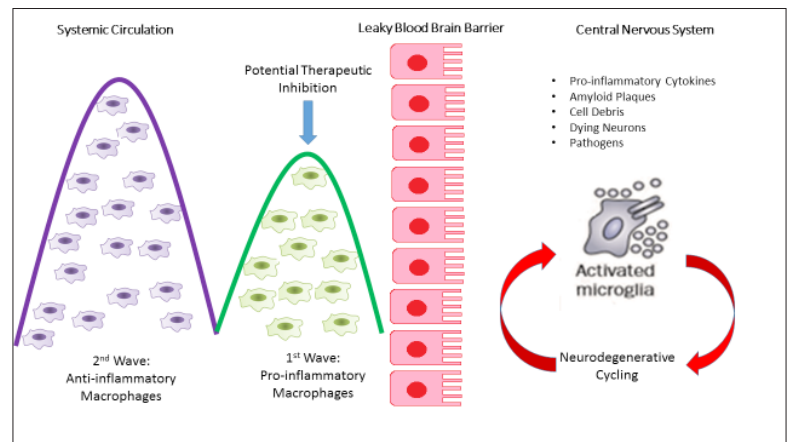

Figure 2: Pathological microglia activation through prolonged systemic inflammation and neurodegenerative cycling and possible immunosuppressive treatment optimization. 


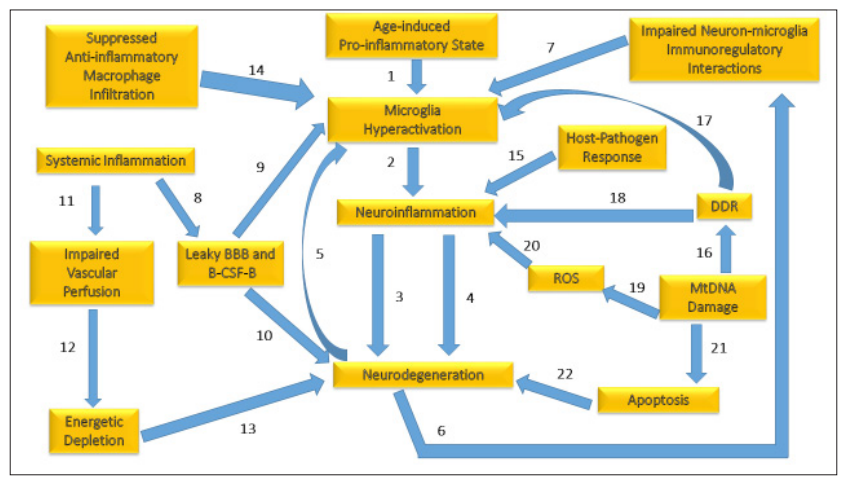

Figure 3: Inflammatory mediators of neurodegeneration.

1. A persistent age-induced proinflammatory state can prime microglia making them hyperactive to immune threats.

2. Microglia hyperactivation can trigger proinflammatory activities that induce neuroinflammation.

3. neuroinflammation

4. generates proinflammatory factors, which contribute to neurodegeneration.

5. Neuroinflammation also stimulates ROS production leading to neurodegeneration.

6. Neurodegeneration in turn can lead to microglia activation via sustained neuron apoptosis, which must be cleared by microglia.

7. Neuron loss from neurodegeneration results in impaired interactions between neurons and microglia, which are important for immunoregulation.

8. Destabilized immunoregulatory interactions between neurons and microglia trigger microglia hyperactivation.

9. Systemic inflammation weakens the tight junctions between the astrocytes and epithelial cells forming the Blood Brain Barrier (BBB) and tight junctions in the epithelial cells forming the Blood-Cerebral Spinal-Fluid Barrier (B-CSF-B) leading to leakiness and vulnerability of the tightly controlled brain environment.

10. A leaky BBB and B-CSF-B allows entry of infiltrating proinflammatory cytokines into the brain microenvironment triggering microglial hyperactivation.

11. A leaky BBB and B-CSF-B also allow infiltrating anti-brain antibodies to contribute to neurodegeneration through interactions with NMDA receptors.

12. Systemic inflammation can damage vasculature and impair perfusion of nutrients to neural cells.

13. Impaired perfusion results in energetic depletion of nutrients and oxygen needed to support costly axonal maintenance.

14. Deficient axonal maintenance results in cell death and neurodegeneration.

15. Suppression of infiltrating anti-inflammatory macrophages, which may happen during immunosuppressive treatment, blocks assistance from the systemic immune system that could downregulate microglia.

16. The host-pathogen response triggers the inflammasome, which activates mediators of proptosis programmed cell death.

17. Mitochondrial DNA damage triggers the DNA Damage Response (DDR).

18. The DDR activates microglia repair genes that stimulate microglia activation.

19. The DDR also activates proinflammatory factors which trigger neuroinflammation.

20. Mitochondrial DNA damage in microglia, when unrepaired, increases ROS.

21. ROS stimulates the release of proinflammatory factors that trigger neuroinflammation.

22. Mitochondrial DNA damage leading to mitochondrial destabilization leads to cytochrome c release, which triggers apoptosis.

23. Apoptosis above normal physiological levels results in neurodegeneration. 


\section{References}

1. Sankowski R, Mader S, Valdes FS (2015) Systemic inflammation and the brain: Novel roles of genetic, molecular and environmental cues as drivers of neurodegeneration. Front Cell Neurosci 9: 28.

2. Schwartz M, Shechter R (2010) Systemic inflammatory cells fight off neurodegenerative disease. Nat Rev Neurol 6(7): 405-410.

3. $\mathrm{Xu} \mathrm{L}, \mathrm{He} \mathrm{D}, \mathrm{Bai} \mathrm{Y}$ (2016) Microglia-mediated inflammation and neurodegenerative disease. Mol Neurobiol 53(10): 6709-6715.

4. Perry VH, Teeling J (2013) Microglia and macrophages of the central nervous system: The contribution of microglia priming and systemic inflammation to chronic neurodegeneration. Semin Immunopathol 35(5): 601-612.

5. Fry M, Ferguson AV (2007) The sensory circumventricular organs: Brain targets for circulating signals controlling ingestive behavior. Physiol Behav 91(4): 413-423.

6. Cunningham C, Wilcockson DC, Campion S, Lunnon K, Perry VH (2005) Central and systemic endotoxin challenges exacerbate the local inflammatory response and increase neuronal death during chronic neurodegeneration. J Neurosci 25(40): 9275-9284.

7. Pott GM, Tarelli R, Ferrari CC, Sarchi MI, Pitossi FJ (2008) Central and systemic IL-1 exacerbates neurodegeneration and motor symptoms in a model of Parkinson's disease. Brain 131(Pt 7): 1880-1894.

8. Busse M, Kunschmann R, Dobrowolny H, Hoffmann J, Bogerts B, et al. (2018) Dysfunction of the blood-cerebrospinal fluid-barrier and N-Methyl-D-Aspartate glutamate receptor antibodies in dementias. Eur Arch Psychiatry Clin Neurosci 268(5): 483-492.

9. Lunnon K, Teeling JL, Tutt AL, Cragg MS, Glennie MJ, et al. (2011) Systemic inflammation modulates Fc receptor expression on microglia during chronic neurodegeneration. J Immunol 186(12): 7215-7224.

10. Lee Y, Morrison BM, Li Y, Lengacher S, Farah MH, et al. (2012) Oligodendroglia metabolically support axons and contribute to neurodegeneration. Nature 487(7408): 443-448.

11. Morrison BM, Lee Y, Rothstein JD (2013) Oligodendroglia: Metabolic supporters of axons. Trends Cell Biol 23(12): 644-651.

12. Kang SH, Li Y, Fukaya M, Lorenzini I, Cleveland DW, et al. (2013) Degeneration and impaired regeneration of gray matter oligodendrocytes in amyotrophic lateral sclerosis. Nat Neurosci 16(5): 571-579.

13. Ferioli M, Zauli G, Maiorano P, Milani D, Mirandola P, et al. (2019) Role of physical exercise in the regulation of epigenetic mechanisms in inflammation, cancer, neurodegenerative diseases, and aging process. J Cell Physiol.

14. Rasley A, Tranguch SL, Rati DM, Marriott I (2006) Murine glia express the immunosuppressive cytokine, interleukin-10, following exposure to Borrelia burgdorferi or Neisseria meningitidis. Glia 53(6): 583-592.

15. Miklossy J (2011) Alzheimer's disease-A neurospirochetosis. Analysis of the evidence following Koch's and Hill's criteria. J Neuroinflammation 8: 90 .

16. Miao EA, Rajan JV, Aderem A (2011) Caspase-1-induced pyroptotic cell death. Immunol Rev 243(1): 206-214.

17. Beckhauser TF, Francis OJ, Pasquale RD (2016) Reactive oxygen species:
Physiological and physiopathological effects on synaptic plasticity. J Exp Neurosci 10(1): 23-48.

18. Dahlgren C, Karlsson A (1999) Respiratory burst in human neutrophils. J Immunol Methods 232(1-2): 3-14.

19. Eisenbarth SC, Flavell RA (2009) Innate instruction of adaptive immunity revisited: The inflammasome. EMBO Mol Med 1(2): 92-98.

20. Gharagozloo M, Mahvelati TM, Imbeault E, Gris P, Zerif E, et al. (2015) The nod-like receptor, Nlrp12, plays an anti-inflammatory role in experimental autoimmune encephalomyelitis. J Neuroinflammation 12: 198.

21. Wang H, Dharmalingam P, Vasquez V, Mitra J, Boldogh I, et al. (2017) Chronic oxidative damage together with genome repair deficiency in the neurons is a double whammy for neurodegeneration: Is damage response signalling a potential therapeutic target? Mech Ageing Dev 161(Pt A): 163-176.

22. Stehling O, Lill R (2013) The role of mitochondria in cellular iron-sulphur protein biogenesis: Mechanisms, connected processes and diseases. Cold Spring Harb Perspect Biol 5(8): a011312.

23. Shen Y, Mackin MZ, Shan Y, Raetz A, David S, et al. (2016) Frataxin deficiency promotes excess microglial DNA damage and inflammation that is rescued by PJ34. PLoS One 11(3): e0151026.

24. Nakanishi H, Hayashi Y, Wu Z (2011) The role of microglial mtDNA damage in age-dependent prolonged LPS-induced sickness behaviour. Neuron Glia Biol 7(1): 17-23.

25. Stephenson J, Nutma E, Valk P, Amor S (2018) Inflammation in CNS neurodegenerative diseases. Immunology 154(2): 204-219.

26. Wang J, Song Y, Chen Z, Leng SX (2018) Connection between systemic inflammation and neuroinflammation underlies neuroprotective mechanism of several phytochemicals in neurodegenerative diseases. Oxid Med Cell Longev 2018: 1972714.

27. Shechter R, London A, Varol C, Raposo C, Cusimano M, et al. (2009) Infiltrating blood-derived macrophages are vital cells playing an antiinflammatory role in recovery from spinal cord injury in mice. PLoS Med 6(7): e1000113.

28. Schneider L, Reichert E, Faulkner J, Reichert B, Sonnen J, et al. (2019) CNS inflammation and neurodegeneration: Sequelae of peripheral inoculation with spinal cord tissue in rat. J Neurosurg: 1-12.

29. London A, Itskovich E, Benhar I, Kalchenko V, Mack M, et al. (2011) Neuroprotection and progenitor cell renewal in the injured adult murine retina requires healing monocyte-derived macrophages. J Exp Med 208(1): 23-39.

30. London A, Benhar I, Mattapallil MJ, Mack M, Caspi RR, et al. (2013) Functional macrophage heterogeneity in a mouse model of autoimmune central nervous system pathology. J Immunol 190(7): 3570-3578.

31. Nakai M, Qin ZH, Chen JF, Wang Y, Chase TN (2000) Kainic acid-induced apoptosis in rat striatum is associated with nuclear factor-kappaB activation. J Neurochem 74(2): 647-658.

32. Fabian RH, Perez PR, Kent TA (2007) A decoy oligonucleotide inhibiting nuclear factor-kappaB binding to the IgGkappaB consensus site reduces cerebral injury and apoptosis in neonatal hypoxic-ischemic encephalopathy. J Neurosci Res 85(7): 1420-1426. 